\title{
Quantum Invariants at the Sixth Root of Unity
}

\author{
Robion Kirby ${ }^{1}$, Paul Melvin ${ }^{2}$, and Xingru Zhang ${ }^{3}$ \\ Department of Mathematics, University of California, Berkeley, CA 94720, USA \\ 2 Department of Mathematics, Bryn Mawr College, Bryn Mawr, PA 19010, USA \\ 3 Department of Mathematics. University of British Columbia, Vancouver, BC V6T 1Y4, Canada
}

Received May 7, 1992

\begin{abstract}
A general topological formula is given for the $S U(2)$ quantum invariant of a 3-manifold $M$ at the sixth root of unity. It is expressed in terms of the homology, Witt invariants and signature defects of the various 2-fold covers of $M$, and thus ties in with basic 4-dimensional invariants. A discussion of the range of values of these quantum invariants is included, and explicit evaluations are made for lens spaces.
\end{abstract}

\section{Introduction}

Quantum invariants of 3-manifolds were introduced by Witten in 1988 using ChernSimons gauge theory and path integrals [W], and subsequently formulated in terms of quantum groups by Reshetikhin and Turaev [RT]. They depend on the choice of a simple compact Lie group (the gauge group) and a root of unity $q$ (of order three or more).

In [KM2], the first two authors established a cabling formula and a symmetry principle for link invariants derived from quantum groups which led to an elementary proof of the existence of the quantum invariants for an $S U(2)$ gauge and to evaluations at the third and fourth roots of unity (in terms of algebraic topological invariants). The existence of such evaluations is not surprising in light of the fact that the $S U(2)$ quantum invariants at $q$ of a 3 -manifold $M_{L}$ obtained by surgery on a framed link $L$ in $S^{3}$ are related to the values of the Jones polynomial of $L$ at $q$, and these values are understood topologically for $q$ of order three or four. In fact, they are also understood at the sixth root of unity, and so it is natural to look for a corresponding evaluation of the quantum invariants. This has been found for 3-manifolds obtained by surgery on a single knot [KM1]. The purpose of this paper is to give a formula for arbitrary closed oriented 3-manifolds $M$. We adopt the notation of [KM2]. In particular, $\tau_{r}(M)$ denotes the $S U(2)$ quantum invariant of $M$ at $q=\exp (2 \pi i / r)$.

Recall from [KM2, Sect. 8.32] that for $r \equiv 2(\bmod 4), \tau_{r}(M)$ splits as a sum of invariants $\tau_{r}(M, \Theta)$ of $M$ equipped with a 1-dimensional cohomology class $\Theta$. (This 
is a consequence of the symmetry principle.) In particular,

$$
\tau_{6}(M)=\sum_{\Theta \in H^{1}(M ; \mathbf{Z} / 2 \mathbf{Z})} \tau_{6}(M, \Theta) .
$$

We shall give a formula for $\tau_{6}(M, \Theta)$ in terms of certain Witt invariants of $M$ and of the 2-fold cover $M_{\Theta} \rightarrow M$ naturally associated with $\Theta$ (by the universal coefficient theorem).

Theorem. Let $M$ be a closed oriented 3-manifold. Then for any element $\Theta$ in $H^{1}(M ; \mathbf{Z} / 2 \mathbf{Z})$,

$$
\tau_{6}(M, \Theta)=\sqrt{3}^{\varepsilon(\Theta)+d\left(M_{\Theta}\right)-d(M)} i^{-w(M)+2 \Theta^{3}+\operatorname{def}_{3}(\Theta)},
$$

where $\varepsilon(\Theta)=0$ or 1 according to whether $\Theta$ is zero or not, $d(M)=\operatorname{rk} H^{1}(M ; \mathbf{Z} / 3 \mathbf{Z})$, $w(M)$ is the mod 3 Witt invariant of $M$ (defined in (6) below), $2 \Theta^{3}$ is the image in $\mathbf{Z} / 4 \mathbf{Z}$ of the cup cube of $\Theta$ (under multiplication by $2: H^{3}(M ; \mathbf{Z} / 2 \mathbf{Z}) \rightarrow H^{3}(M ; \mathbf{Z} / 4 \mathbf{Z})$ ), and $\operatorname{def}_{3}(\Theta)$ is the mod 3 Witt defect of the 2-fold cover $M_{\Theta} \rightarrow M$ (defined in (9)).

Note that if $M$ is a $\mathbf{Z} / 2 \mathbf{Z}$-homology sphere, then $\tau_{6}(M)=\tau_{6}(M, 0)$, and the formula in the theorem reduces to

$$
\tau_{6}(M)=\sqrt{3}^{d(M)} i^{-w(M)},
$$

since $M_{\Theta}$ is just two copies of $M$. In particular, $\tau_{6}(M)=1$ for any integral homology sphere $M$.

\section{Witt Invariants and Defects}

For any integral symmetric bilinear form $A$, let $\sigma_{A}$ denote the signature of $A$, and let $d_{A}$ and $w_{A}$ denote the nullity and Witt class of $A$ viewed as a form over $\mathbf{Z} / 3 \mathbf{Z}$ (lying respectively in $\mathbf{Z}$ and in the Witt group $W(\mathbf{Z} / 3 \mathbf{Z})=\mathbf{Z} / 4 \mathbf{Z}$, see for example $[\mathrm{MH}])$. Recall that $w_{A}$ can be computed by diagonalizing $A(\bmod 3)$, with diagonal entries written as 0 or \pm 1 , and then taking the trace. Set

$$
\lambda_{A}=\sqrt{3}^{d_{A}} i^{-w_{A}} \text {. }
$$

This expression arises in the evaluation of Jones polynomials at the sixth root of unity. In particular, for any oriented link $L$ in $S^{3}$, let $Q(L)$ denote the quadratic form of $L$ (obtained by taking the sum of the Seifert form and its transpose). Then

$$
\tilde{V}_{L}=\lambda_{Q(L)}
$$

by Theorem (B.1) of [KM2], where $\tilde{V}$ is the variant of the Jones polynomial defined in [KM2, Sect. 4].

If $L$ is a framed link, then there is another symmetric bilinear form associated with $L$, the linking form $A(L)$ with framings down the diagonal. We shall write $\sigma_{L}, d_{L}$, $w_{L}$, and $\lambda_{L}$, respectively, for $\sigma_{A(L)}, d_{A(L)}, w_{A(L)}$, and $\lambda_{A(L)}$.

Following [KM2], denote the colored framed link invariant of $L$ with coloring $\mathbf{k}$ at $q=\exp (2 \pi i / 6)$ by $J_{L, \mathbf{k}}$, and write $J_{L}$ for $J_{L, 2}$. Note that $J_{L}$ is independent of the orientation on $L$. Applying Corollary (4.11) of [KM2] at the sixth root of 1 and Eq. (4), we have (for any orientation on $L$ )

$$
J_{L}=[2] t^{3 L \cdot L} \tilde{V}_{L}=\sqrt{3} \zeta^{L \cdot L} \lambda_{Q(L)},
$$

where $\zeta=\exp (2 \pi i / 8)$ (since [2] $=\sqrt{3}$ and $t=\exp (2 \pi i / 24))$. 
Now let $M$ be a closed oriented 3-manifold. Choose any compact oriented 4manifold $W$ bounded by $M$, with intersection form $A(W)$, and define the mod 3 Witt invariant of $M$ by

$$
w(M)=\sigma_{A(W)}-w_{A(W)}(\bmod 4) .
$$

(In particular $w\left(M_{L}\right)=\sigma_{L}-w_{L}$.) To see that this definition is independent of the choice of $W$, note that the signature and the Witt class agree $(\bmod 4)$ for closed 4-manifolds since their intersection forms are stably diagonalizable over the integers (i.e. allow block sums with $(1) \oplus(-1))$. The invariance of $w(M)$ now follows from Novikov additivity of the signature and Witt class. (The latter is immediate from the proof in [AS, Sect. 7] which shows that the intersection form of the closed 4-manifold obtained by gluing $W$ and $W^{\prime}$ along their common boundary is Witt equivalent to the sum of the nondegenerate parts of the forms of $W$ and $W^{\prime}$.) There is a similar definition for the $\bmod p$ Witt invariants for any prime $p \equiv 3(\bmod 4)$.

Next consider an $m$-fold cyclic cover $\tilde{M} \rightarrow M$ of closed oriented 3-manifolds. This extends to a cyclic cover $\tilde{W} \rightarrow W$ of compact oriented 4-manifolds, branched along a closed surface $F$ in $W$ (see for example [CG, Lemma 2.2]). Denote the intersection forms of $W$ and $\tilde{W}$ by $A(W)$ and $A(\tilde{W})$.

Hirzebruch's signature defect of the cover $\tilde{M} \rightarrow M[\mathrm{H}]$ (see also [KM3, Sect.3]) is defined to be the rational number

$$
\operatorname{def}(\tilde{M} \rightarrow M)=m \sigma_{A(W)}-\sigma_{A(\tilde{W})}-\frac{m^{2}-1}{3 m} F \cdot F .
$$

This is independent of the choice of the cover $\tilde{W} \rightarrow W$ by Novikov additivity and the $G$-signature theorem. Note that $F \cdot F$ is divisible by $m$, and so $3 \operatorname{def}(\tilde{M} \rightarrow M)$ is an integer.

Similarly, define the mod 3 Witt defect of the cover $\tilde{M} \rightarrow M$ by

$$
\operatorname{def}_{3}(\tilde{M} \rightarrow M)=m w_{A(W)}-w_{A(\tilde{W})}-\frac{m^{2}-1}{3 m} F \cdot F(\bmod 4) .
$$

This is well defined by Novikov additivity and the fact that the signature reduces $\bmod 4$ to the Witt class for closed 4-manifolds. There is an analogous definition for the $\bmod p$ Witt defects of covers for primes $p \equiv 3(\bmod 4)$.

Question. Is $\operatorname{def}_{3}(\tilde{M} \rightarrow M)$ a homotopy invariant? That is, if $\tilde{M} \rightarrow M$ is the pull back of $\tilde{N} \rightarrow N$ under a homotopy equivalence $M \rightarrow N$, then is $\operatorname{def}_{3}(\tilde{M} \rightarrow$ $M)=\operatorname{def}_{3}(\tilde{N} \rightarrow N)$ ? (The answer is probably yes. If so, then $\tau_{6}(M . \Theta)$ and $\tau_{6}(M)=\sum \tau_{6}(M, \Theta)$ are homotopy invariants as well.)

Now for any closed oriented 3-manifold $M$ and class $\Theta$ in $H^{1}(M ; \mathbf{Z} / 2 \mathbf{Z})$, define

$$
\operatorname{def}_{3}(\Theta)=\operatorname{def}_{3}\left(M_{\Theta} \rightarrow M\right),
$$

where $M_{\Theta} \rightarrow M$ is the associated 2-fold cover.

Remark. The corresponding signature defect $\operatorname{def}(\Theta)$ is an integer (since $m=2$ ), and is related to $\operatorname{def}_{3}(\Theta)$ by the congruence $\operatorname{def}_{3}(\Theta)=\operatorname{def}(\Theta)-\left(2 w(M)-w\left(M_{\Theta}\right)\right)$ $(\bmod 4)$. Thus the formula in the theorem can be rewritten as

$$
\tau_{6}(M, \Theta)=\sqrt{3}^{\varepsilon(\Theta)+d\left(M_{\Theta}\right)-d(M)} i^{w(M)+w\left(M_{\Theta}\right)+2 \Theta^{3}+\operatorname{def}(\Theta)} .
$$

The preceding question asks whether or not the signature defect $(\bmod 4)$ is a homotopy invariant. 
If $M=M_{L}$, then $\Theta$ corresponds to a sublink $E$ and $L$ (consisting of those components whose meridians $\mu$ have $\Theta(\mu) \neq 0$ ) and the self linking number $E \cdot E$ (i.e. the sum of all the entries in the linking matrix $A(E)$ ) is even.

If $E$ is oriented, then it corresponds to an integral 2-dimensional homology class in the 4-manifold $W_{L}$ obtained by adding 2-handles to $B^{4}$ along $L$. This class can be represented, for example, by the surface $F_{E}$ consisting of the cores of all the handles attached to the components of $E$ together with a Seifert surface for $E$ (with interior pushed into $B^{4}$ ).

Note that the self intersection number of $F_{E}$ is equal to the self linking number of $E$ which is clearly independent $\bmod 4$ of the orientation on $E$. In fact, it reduces $\bmod 4$ to the element $2 \Theta^{3}$ in $\mathbf{Z} / 4 \mathbf{Z}$ :

$$
F_{E} \cdot F_{E}=E \cdot E \equiv 2 \Theta^{3}(\bmod 4) .
$$

One way to see this congruence is by Poincaré duality. Indeed, one must show that the triple self intersection of the dual of $\Theta$ (which is dual to $\Theta^{3}$ ) has the same parity as $e=\frac{1}{2} E \cdot E$. This is easiest to verify by first "sliding handles" and "blowing up" so that $E$ consists of a single unknotted component with framing $2 e$. Then the dual of $\Theta$ is represented by a Seifert surface for $E$ (disjoint from $L-E$ ) capped off with a punctured sum of $e$ projective planes in the surgery solid torus, and the triple self intersection of this surface is $e(\bmod 2)$.

Now observe that the two fold cover $\tilde{W}$ of $W_{L}$ branched along $F_{E}$ is bounded by the cover $M_{\Theta} \rightarrow M$. Thus for any framed link $\tilde{L}$ for which $W_{\tilde{L}}=\tilde{W}$, we have

$$
\operatorname{def}_{3}(\Theta)=2 w_{L}-w_{\tilde{L}}-e(\bmod 4)
$$

where $e=\frac{1}{2} E \cdot E$.

\section{Proof of the Theorem}

As above, we adopt the notation of [KM2]. Choose a framed link $L$ in $S^{3}$ with $M=M_{L}$ and let $E$ be the sublink corresponding to the cohomology class $\Theta$. Note that $E \cdot K$ is even for each component $K$ of $L$ (by Poincaré duality). Denote the complementary sublink $L-E$ by $E^{\prime}$. Then

$$
\tau_{6}\left(M_{L}, \Theta\right)=\frac{i^{-\sigma_{L}}}{\sqrt{3} n_{E^{\prime}}} \sum_{S<E^{\prime}} J_{E \cup S, \mathbf{2} \cup \mathbf{3}},
$$

where $n_{L}$ denotes the number of components in $L$. This is just Theorem (8.32) in [KM2] for $r=6$.

By the cabling formula [KM2, Theorem (4.15) and the following remark] (at the sixth root of 1)

$$
J_{E \cup S, \mathbf{2} \cup \mathbf{3}}=\sum_{T<S}(-1)^{n_{S}-T} J_{E \cup 2 T} .
$$

(Here $2 T$ denotes the double cable of $T$.)

Now for any given sublink $T<E^{\prime}$, there are $2^{n} E^{\prime}-T$ sublinks $S$ with $T<S<E^{\prime}$. In particular, substituting (14) in (13) and interchanging the order of summation we 
obtain

$$
\begin{aligned}
\tau_{6}\left(M_{L}, \Theta\right) & =\frac{i^{-\sigma_{L}}}{\sqrt{3}^{n_{E^{\prime}}}} \sum_{T<E^{\prime}} \sum_{T<S<E^{\prime}}(-1)^{n_{S}-T} J_{E \cup 2 T} \\
& =\frac{i^{-\sigma_{L}}}{\sqrt{3}^{n_{E^{\prime}}}} \sum_{T<E^{\prime}} \sum_{j=0}^{n_{E^{\prime}-T}}(-1)^{j}\left(\begin{array}{c}
n_{E^{\prime}-T} \\
j
\end{array}\right) J_{E \cup 2 T} \\
& =\frac{i^{-\sigma_{L}}}{\sqrt{3}^{n_{E^{\prime}}}} J_{E \cup 2 E^{\prime}}
\end{aligned}
$$

since the alternating sum of the entries in any row but the first of Pascal's triangle is zero.

Using (3) and (5) it follows from (15) that

$$
\tau_{6}\left(M_{L}, \Theta\right)=\sqrt{3}^{1+d_{Q}-n_{E^{\prime}}} i^{e-\sigma_{L}-w_{Q}}
$$

for any chosen orientation on $L$, where $e=\frac{1}{2} E \cdot E$ (dual to $\Theta^{3}$ by (11)) and $Q=Q\left(E \cup 2 E^{\prime}\right)$.

In practice, formula (16) provides the easiest computation of $\tau_{6}$. But to show how it is related to the Witt invariants discussed above, it is convenient to consider separately the two cases when $\Theta$ is trivial or not.

If $\Theta=0$, then $E$ is empty, and so (16) reduces to

$$
\tau_{6}\left(M_{L}, 0\right)=\sqrt{3}^{1+d_{Q}-n_{L}} i^{-\sigma_{L}-w_{Q}},
$$

where $Q$ is the quadratic form of $2 L$. Now $2 L$ (with each doubled pair oppositely oriented) has a Seifert surface obtained by replacing each component of $L$ by an annulus, twisted according to the framing, and then connecting these annuli with $\left(n_{L}-1\right)$ tubes. The resulting Seifert matrix is $O \oplus A_{L}$ (where $O$ is a $\left(n_{L}-1\right) \times\left(n_{L}-1\right)$ matrix of 0's) and so the associated quadratic form $Q$ is $O \oplus\left(A_{L}+A_{L}^{t}\right)=O \oplus 2 A_{L} \equiv$ $O \oplus-A_{L}(\bmod 3)$. Thus $d_{Q}=n_{L}-1+d_{L}$ and $w_{Q}=-w_{L}$ and the formula in the theorem follows immediately.

If $\Theta \neq 0$, then we can arrange that $E=L$. (If not, then slide one component of $E$ over each of the components of $E^{\prime}$.) Now (16) reduces to

$$
\tau_{6}\left(M_{L}, \Theta\right)=\sqrt{3}^{1+d_{Q}} i^{e-\sigma_{L}-\omega^{\prime} Q},
$$

where $Q$ is the quadratic form of $L$, and so it remains to prove

$$
d_{Q}=d\left(M_{\Theta}\right)-d(M) \text { and } w_{Q}=w(M)-e-\sigma_{L}-\operatorname{def}_{3}(\Theta) .
$$

To establish (19), we will explicitly construct the double branched cover $W_{\tilde{L}}$ of $W_{L}$ described above (12), which is bounded by the double cover $M_{\theta}$ of $M$.

First orient $L$ and choose a Seifert surface $F$ in the 3 -sphere. A framed link $L^{\prime}$ for the double cover of the 4-ball branched along $F$ (with its interior pushed in $B^{4}$ ) is described in [AK, Sect. 2]:

View $F$ as a 2-disc $D$ with bands (1-handles) attached, as in Fig. 1a. Let $\varrho$ denote a rotation of $S^{3}$ about the circle $\partial D$ by $\pi$ radians which carries the interior of $D$ off itself. We may assume that the bands of $F$ all lie close to the disc $\varrho(D)$ and are disjoint from their images under $\varrho$, which lie close to $D$. Now $L^{\prime}$ consists of the knots obtained by joining the cores of the bands with their images under $\varrho$, and the framing on each component is given by the number of half twists in the associated 


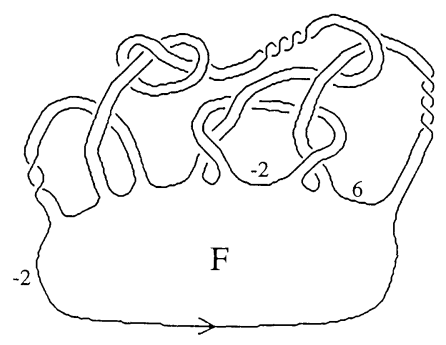

(a)

Fig. 1a, b

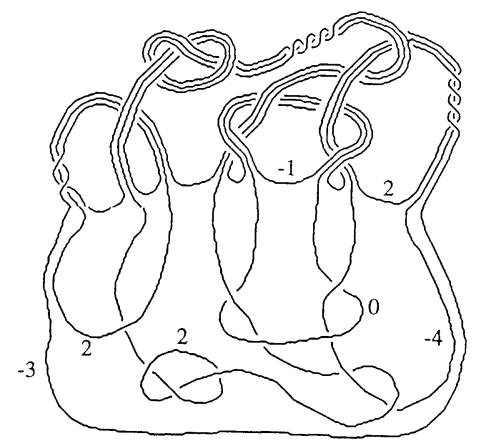

(b)

band. For example, the band in Fig. 1a with the trefoil knot tied in it has 6 right half twists, from the knot, and 4 additional left half twists, and so is assigned the framing 2 as in Fig. $1 b$.

Now let $F_{L}$ denote the closed surface in $M_{L}$ obtained from $F$ (pushed into $B^{4}$ ) by capping off the boundary circles with the cores of the 2-handles attached to $L$. The desired double cover of $W_{L}$, branched along $F_{L}$, is obtained by adding 2-handles to $W_{L^{\prime}}$ along the lift of $L=\partial F$, and so is given by the link

$$
\tilde{L}=L \cup L^{\prime}
$$

with modified framings on $L$.

To determine the new framings, observe that each component $K$ of $L$ has two natural framings, one as a component of $L$ (the $L$-framing $k$ ) and the other as a curve in $F$ (the $F$-framing $f$, which is the linking number with a pushoff in $F$ ). The new framing on $K$ is just the average $(k+f) / 2$ of these two framings - this is an integer since $L \cdot K$ is even. To see this, note that the 2-handle attached to $K$ in $M_{\tilde{L}}$ is the double cover of the corresponding one in $M_{L}$, branched along its core, and so the relative framing $k-f$ must be halved. Thus the new framing is $f+(k-f) / 2=(k+f) / 2$. For example, the component with $L$-framing 6 in Fig. 1a has $F$-framing -2 , and so has framing 2 in the cover as shown in Fig. 1 b.

We now claim that the linking matrix of $\tilde{L}$ is equivalent $\bmod 3$ to the block sum of the negative of the linking matrix of $L$ and the symmetrized Seifert matrix of $F$ (which represents $Q=Q(L)$ ):

$$
A(\tilde{L}) \equiv-A(L) \ominus Q(L)(\bmod 3) .
$$

To prove (20), note that there are $2 g+n-1$ bands in $F$, where $g$ is the genus of $F$ and $n=n_{L}$ is the number of components in $L$. Label them (arbitrarily) with the letters $a_{1}, b_{1}, \ldots, a_{g}, b_{g}, c_{1}, \ldots, c_{n-1}$, and then (for convenience) slide them over one another so that they are attached in the order $a_{1} b_{1} a_{1} b_{1} \ldots a_{g} b_{g} a_{g} b_{g} c_{1} c_{1} \ldots c_{n-1} c_{n-1}$.

Let $A$ denote the linking matrix for the bands $a_{1}, b_{1}, \ldots, a_{g}, b_{g}$ (i.e. the $i j^{\text {th }}$ entry records the number of half twists between the $i^{\text {th }}$ and $j^{\text {th }}$ band). The corresponding linking matrix for the bands $c_{1} \ldots \ldots c_{n-1}$ has even entries, since $F$ is orientable, and so will be denoted by $2 B$. Similarly, $2 C$ will denote the matrix which records the linking between the first $2 g$ bands and the last $n-1$. Finally, let $2 a$ denote the difference of the $L$-framing and the $F$-framing of the "long" component of $L$ (the one which passes over every band of $F$ ), and let $2 D$ denote the corresponding diagonal matrix for the framing differences of the remaining components of $L$ (corresponding to the bands $c_{1}, \ldots, c_{n-1}$ ). 
Now it is easily verified that the linking matrix and quadratic form for $L$ are given by

$$
A(L)=\left(\begin{array}{cc}
s+2 a & b^{t} \\
b & B+2 D
\end{array}\right) \text { and } Q(L)=\left(\begin{array}{cc}
A & 2 C \\
2 C^{t} & 2 B
\end{array}\right) .
$$

where $s$ is the sum of all the entries of $B$ and $b$ is the column vector whose $i^{\text {th }}$ entry is the sum of the entries in the $i^{\text {th }}$ row of $B$. Similarly, the linking matrix for $L$ is of the following block form:

$$
A(\tilde{L})=\left(\begin{array}{cccc}
s+a & b^{t} & c^{t} & b^{t} \\
b & B+D & C^{t} & B \\
c & C & A & 2 C \\
b & B & 2 C^{t} & 2 B
\end{array}\right),
$$

where $c$ is the column vector whose $i^{\text {th }}$ entry is the sum of the entries in the $i^{\text {th }}$ row of $C$. A change of basis converts $A(\tilde{L})$ into

$$
\left(\begin{array}{cccc}
5 s+a & 5 b^{t} & 3 c^{t} & 3 b^{t} \\
5 b & 5 B+D & 3 C^{t} & 3 B \\
3 c & 3 C & A & 2 C \\
3 b & 3 B & 2 C^{t} & 2 B
\end{array}\right)
$$

which is congruent to $-A(L) \oplus Q(L)(\bmod 3)$. In particular, first add the last block of rows (and then columns) to the second block, and then add each row (column) in the last block to the first row (column). This establishes (20).

It follows that $d_{Q}=d_{\tilde{L}}-d_{L}=d\left(M_{\Theta}\right)-d(M)$ (since $A(L)$ and $A(\tilde{L})$ are presentation matrices for the first homology of $M$ and $M_{\Theta}$ ), which proves the first equation of (19). Also $w_{Q}=w_{\tilde{L}}+w_{L}$, which gives the second equation of (19) using (6) and (12), and the theorem is proved.

Remark. The invariant $\Theta^{3}$ is determined by the other invariants which appear in the exponents in the formula in the theorem. In particular,

$$
\Theta^{3} \equiv d\left(M_{\Theta}\right)+\operatorname{def}_{3}(\Theta)+\varepsilon(\Theta)(\bmod 2) \text {. }
$$

This is obvious if $\varepsilon(\Theta)=0$ (i.e. $\Theta=0$ ), since all the terms in (24) except $d\left(M_{\Theta}\right)$ then vanish, and $d\left(M_{\Theta}\right)=2 d(M)$. If $\varepsilon(\Theta)=1$ then the link $\tilde{L}$ constructed in the proof of the theorem has an odd number of components by (22), and so $d\left(M_{\Theta}\right)=$ $d_{\tilde{L}} \equiv w_{\tilde{L}}+1(\bmod 2)$. Thus using $(12)$ we have $\Theta^{3} \equiv e \equiv 2 w_{L}-w_{\tilde{L}}+\operatorname{def}_{3}(\Theta) \equiv$ $d\left(M_{\Theta}\right)+\operatorname{def}_{3}(\Theta)+1$.

\section{Examples: Lens Spaces}

Simple formulas for the quantum invariants of lens spaces $L(c, a)$ (with $c \neq 0$ ) at the sixth root of unity can be derived using (16) and the techniques of [KM3]. In particular, adopting the orientation conventions of [KM3],

$$
\tau_{6}(L(c, a), 0)= \begin{cases} \pm 1 & \text { for } c \equiv \pm 1(\bmod 3) \\ \pm \sqrt{3} i & \text { for }(c, a) \equiv(0, \pm 1)(\bmod 3)\end{cases}
$$

and for $c$ even and $\Theta \neq 0$,

$$
\tau_{6}(L(c \cdot a) . \Theta)= \begin{cases} \pm \sqrt{3} & \text { for } c / 2 \equiv \pm 1(\bmod 4) \\ \pm \sqrt{3} i & \text { for }(c / 2 \cdot a) \equiv(0 . \pm 1) \text { or }(2, \mp 1)(\bmod 4) .\end{cases}
$$


(For $c=0$ one readily computes $\tau_{6}\left(S^{2} \times S^{1} . \Theta\right)=\sqrt{3}$ for either class $\Theta$.)

We will prove these formulas by induction on $c$. For convenience, assume that $a / c$ is a Farey fraction (i.e. $a$ and $c$ are coprime and $0 \leq a \leq c$ ), and so $0 \leq a / c \leq 1$. The induction starts with the case of the 3 -sphere $(c=1$, or equivalently $a / c=0$ or 1) which is trivially verified. Now assume $c>1$, and so $0<a / c<1$.

Recall that $L(c, a)$ can be described by surgery on any simple chain link $L$ with framings $a_{1}, \ldots, a_{n}$ chosen so that $a / c=\left\langle a_{1}, \ldots, a_{n}\right\rangle$, as shown in Fig. 2. Here, as in $[\mathrm{KM} 3],\left\langle a_{1}, \ldots, a_{n}\right\rangle$ denotes the continued fraction $-1 /\left(a_{1}-1 /\left(a_{2}-\right.\right.$ $\left.\left.\ldots-1 / a_{n}\right) \ldots\right)$. Geometrically, the list $a_{1}, \ldots, a_{n}$ defines an ideal edge path in the hyperbolic plane, with vertices $\propto .0 .\left\langle a_{1}\right\rangle,\left\langle a_{1}, a_{2}\right\rangle \ldots .\left\langle a_{1}, \ldots a_{n}\right\rangle=a / c$ on the circle at infinity [KM3, Sect. 1]. Each component of $L$ corresponds to an interior vertex of this path.

Fig. 2
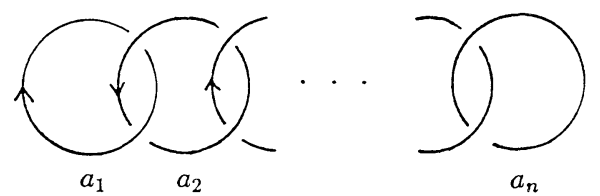

By the inductive assumption, $L$ can be chosen with $n>1$ so that the last two interior vertices of the associated path, $p / q=\left\langle a_{1}, \ldots, a_{n-2}\right\rangle$ and $\mathrm{r} / \mathrm{s}=$ $\left\langle a_{1} \ldots \ldots a_{n-1}\right\rangle$, are Farey fractions with $p / q<a / c<r / s$ and $p s=q r-1$. (Here $p / q=0 / 1$ if $n=2$.) In particular, $q$ and $s$ are both less than $c$, and so by induction the formulas $(25,26)$ hold (if applicable) for $L(q \cdot p)$ and $L(s, r)$.

First let $\Theta=0$. Note that in general

$$
\tau_{6}(M .0)=\sqrt{3}^{d(M)} i^{-u^{(M)}},
$$

and so $\left|\tau_{6}(L(c, a), 0)\right|=\sqrt{3}$ or 1 , according to whether $c$ is divisible by 3 or not. Thus we need only analyze the phase in formula (25).

Choose $L$ so that $a_{n}=1$, and so $(c \cdot a)=(q+s, p+r)$. Then

$$
w(L(c, a))=w(L(s, r))+ \begin{cases}0 & \text { if } q \equiv 0(\bmod 3) . \\ 2 & \text { if } s \equiv q(\bmod 3) . \\ 3 & \text { otherwise }\end{cases}
$$

Indeed, $L(s, r)$ is given by the framed link $J$, obtained from $L$ by dropping the last component. In passing from $J$ to $L$, the signature of the linking form increases by 1 (use $[\mathrm{KM} 3,(1.17 \mathrm{c})]$ ), while the Witt class increases by 1 if $q \equiv 0$, decreases by 1 if $s \equiv q(\bmod 3)$, and remains fixed otherwise (diagonalize from the top down and use $[\mathrm{KM} 3,(1.13 \mathrm{~b})$ and $(1.17 \mathrm{c})])$. The Witt invariant is the difference of the signature and the Witt class. Formula (25) now follows by induction, using the fact that $p s \equiv q r-1(\bmod 3)$.

Now suppose that $c$ is even and $\Theta \neq 0$. Let $L_{0}$ denote the sublink of $L$ corresponding to the vertices $p / q$ of type 0 (i.e. $(p, q) \equiv(0.1)(\bmod 2))$ in the associated path, and write $n_{0}$ for the number of components in $L_{0}$ and $\tau_{0}=L_{0} \cdot L_{0}$ for the sum of the framings. Similarly, define $L_{1}, n_{1}, \tau_{1}$ for vertices of type 1 (三(1.1)), and $L_{\infty}, n_{\infty}, \tau_{\infty}$ for those of type $\propto(\equiv(1,0))$. Note that $a / c$ is of type $\propto$.

Observe that the characteristic sublink $E$ corresponding to $\Theta$ is just $L_{0} \cup L_{1}$ (cf. the proof of (4.6) in [KM3]), and $E^{\prime}=L_{x}$. Thus $n_{E^{\prime}}=n_{x}$. To analyze the other terms in (16), orient the components of $L$ alternately clockwise and counterclockwise, 
as shown in Fig. 2. Then $e=\frac{1}{2} E \cdot E=\frac{1}{2}\left(\tau_{0}+\tau_{1}\right)+n-1-2 n_{\infty}$. An easy calculation shows that the quadratic form $Q=Q\left(E \cup 2 L_{\infty}\right)$ is Witt equivalent to $O_{n_{x}} \otimes I_{n-1-2 n_{\infty}}$, and so $d_{q}=n_{\infty}$ and $w_{Q}=n-1-2 n_{\infty}$. Putting these together in (16) gives

$$
\tau_{6}(L(c . a) . \Theta)=\sqrt{3} i^{\frac{1}{2}\left(\tau_{0}+\tau_{1}\right)-\sigma_{L}}=\sqrt{3} i^{\mu_{0}+\mu_{1}},
$$

where $\mu_{0}$ and $\mu_{1}$ are the two mu invariants of $L(c, a)$ [KM3, Sect. 4].

It follows from (27) that $\left|\tau_{6}(L(c . a), \Theta)\right|=\sqrt{3}$. Since (26) can be rewritten

$$
\tau_{6}(L(c, a), \Theta)=\sqrt{3} i \begin{cases}c / 2+a & \text { if } c \equiv 0(\bmod 4), \\ c / 2-1 & \text { if } c \equiv 2(\bmod 4)\end{cases}
$$

it remains to show that the exponents of $i$ in (27) and (28) are equal.

Note that $L$ can be chosen so that either $q$ is even and $a_{n}=2$, or $s$ is even and $a_{n}=-2$. We assume the former, as the arguments are analogous. Thus $(c, a)=(q+2 s \cdot p+2 r)$, and $(26)$ is assumed to hold for $L(q, p)$.

Now $L(q \cdot p)$ is given by the framed link $J$ obtained from $L$ by dropping the last two components. In passing from $J$ to $L$, the signature of the linking form remains unchanged (again use $[\mathrm{KM} 3,(1.17 \mathrm{c})]$ ) and the invariant $\tau_{0}+\tau_{1}$ increases by 2. Thus the exponent of $i$ in (27) increases by 1 . The same is true in (28). Indeed, $(c / 2, a)=(q / 2, p)+(s, 2 r)$, and so the exponent of $i$ in (28) increases by $p+s+2 r+1$ if $c \equiv 0(\bmod 4)$, and by $s-p-1$ if $c \equiv 2(\bmod 4)$. (Note that $q \equiv c+2(\bmod 4))$. But both of these expressions equal 1 , since $p s \equiv q r-1(\bmod 4)$. This completes the proof of (26).

\section{Range of Values}

Using the arguments in the proof of the theorem, one can determine the full range of values of the invariants $\tau_{6}(M, \Theta)$, and more generally of the invariants which appear in the exponents in the formula given in the theorem. For convenience, denote the list $(\varepsilon \cdot d, \bar{d} \cdot w \cdot \delta)=\left(\varepsilon(\Theta), d(M), d\left(M_{\Theta}\right), w(M), \operatorname{def}_{3}(\Theta)\right)$ of invariants of $(M . \Theta)$ by $\sigma=\sigma(M, \Theta)$. (Note that we have excluded $\Theta^{3}$ since it is determined by the other invariants, by the remark above.) Also set

$$
\mathcal{F}=\left\{\sqrt{3}^{s} i^{t} \mid s \in \mathbf{Z}, t \in \mathbf{Z} / 4 \mathbf{Z}, s \geq 0\right\} .
$$

Proposition. The invariants $\sigma=(\varepsilon . d, \bar{d}, w, \delta)$ assume all values in $\mathbf{Z} / 2 \mathbf{Z} \ominus \mathbf{Z} \ominus \mathbf{Z} \ominus$ $\mathbf{Z} / 4 \mathbf{Z} \bigcirc \mathbf{Z} / 4 \mathbf{Z}$ satisfying the following conditions:

(i) $\bar{d} \geq d \geq 0$,

(ii) if $\bar{d}=0$, then $w \equiv 0(\bmod 2)$, and

(iii) if $\subseteq=0$ (i.e. $\Theta=0$ ), then $\bar{d}=2 d$ and $\delta=0$.

The full range of values of $\tau_{6}(M, 0)$ is the set,$-\{ \pm i\}$, and of $\tau_{6}(M, \Theta)$ (for $\Theta \neq 0$ ) is the set $Y \pm 1, \pm i\}$.

Proof. Necessity of the conditions is easy: (i) follows from (19), (ii) follows from the fact that $\sigma_{L} \equiv w_{L}(\bmod 2)$ when $d(M)=0$, and (iii) is obvious.

Sufficiency is proved by construction. It suffices to (a) produce examples of $(M, \Theta)$ with $\sigma=(\varepsilon, 0,0, u ; \varepsilon \delta)$ for arbitrary $\varepsilon$ and $\delta$ and even $w$, (b) show how to modify any $(M, 0)$ to change $\sigma$ by $(0,1,2, w, 0)$ for $w=0$ or 1 , and (c) show how to modify 
any $(M, \Theta)$ with $\Theta \neq 0$ to change $\sigma$ by $(0,0,1,0,-1)$, and by $(0,1,1, w,-1)$ for $w=0$ or 1 .

The lens spaces $L(c, 1)$ (obtained by surgery with framing $-c$ on the unknot) provide examples for (a). In particular, using (16) one computes

$$
\sigma(L(6 k \pm 2,1), \Theta)=(\varepsilon(\Theta) .0,0,1 \pm \operatorname{sign}(k),-\varepsilon(\Theta) k)
$$

for $k \neq 0$, and so the values $0<k \leq 4$ suffice.

To achieve (b), one may take connected sums with $(L(3 k, 1), 0)$ for $k=0$ or -1 . Indeed, using (16) one computes $\sigma(L(3 k, 1), 0)=(0,1,2,-\operatorname{sign}(k), 0)$, and in general

$$
\sigma\left((M, \Theta) \leftleftarrows\left(M^{\prime}, \Theta^{\prime}\right)\right)=\sigma(M . \Theta)+\sigma\left(M^{\prime} . \Theta^{\prime}\right)+\left(-\varepsilon \varepsilon^{\prime}, 0, \varepsilon \varepsilon^{\prime}, 0,0\right),
$$

where $\varepsilon=\varepsilon(M, \Theta)$ and $\varepsilon^{\prime}=\varepsilon\left(M^{\prime}, \Theta^{\prime}\right)$.

For (c), let $(M, \Theta)$ be given by a framed link $L$ with sublink $E$ corresponding to $\Theta$ (as above). Consider the operation of adjoining a doubled meridian with framing $k$ to a component of $E$, as shown in Fig. 3 .

Fig. 3

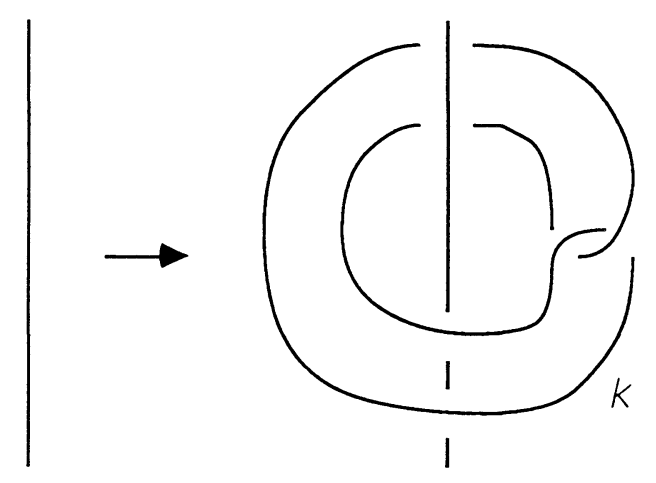

This has the effect of block summing the linking matrix of $L$ with $(k)$, and the quadratic form of $L$ with $(1-k) \oplus(0)$ (up to Witt equivalence). From this one easily computes the effect on $\sigma$ using (16). In particular, $\sigma$ changes by $(0,0,1,0,-1)$ for $k=1$, by $(0,1,1,0,-1)$ for $k=0$, and by $(0,1,1,1,-1)$ for $k=3$.

The last statement in the proposition follows immediately (or can be proved directly).

Remark. It is not so easy to determine the full range. $\mathcal{T}$ of values of $\tau_{6}(M)=$ $\sum \tau_{6}(M, \Theta)$. The proposition shows that $\widetilde{T}$ is a multiplicative subset of the ring $\Lambda=\mathbf{Z}[\sqrt{3}, \sqrt{3} i]$, but it appears difficult to characterize this subset. It is not hard to show that $\mathcal{T}$ is a proper ubset of $\Lambda$. For example, any $a+b \sqrt{3}+c \sqrt{3} i+3 d i$ in $\mathscr{T}-\mathcal{Y}$ (for $a, b, c$, and $d$ integers and ' $/$ as in the proposition) must have $a+b+c+d$ even. Also, $\mathcal{T}$ is not discrete as a subset of $\mathbf{C}$. For example, $\tau_{6}\left(\mathbf{R} P^{3}\right)=\sqrt{3}-1$ and $\tau_{6}(L(12,1))=0$ (see above for the computation for lens spaces) and so the infinite sequence $\tau_{6}\left(n \mathbf{R} P^{3}\right)$ converges to $\tau_{6}(L(12,1))$. Is . $\mathcal{T}$ dense in $\mathbf{C}$ ? 


\section{References}

[AK] Akbulut, S., Kirby, R.: Branched covers of surfaces in 4-manifold. Math. Ann. 252, 111-131 (1980)

[AS] Atiyah, M.F., Singer, I.M.: The index of elliptic operators. IIl. Ann. Math. 87, 546-604 (1968)

[CG] Casson, A.J., Gordon, C.McA.: On slice knots in dimension three. In: Geometric Topology. Proc. Symp. Pure Math. XXXII, Providence, RI: Am. Math. Soc. 1976, pp. 39-53

[H] Hirzebiuch, F.: The signature theorem: Reminiscences and recreation. In: Prospects in Math. Ann. Math. Studies 70, Princeton, NJ: Princeton Univ. Press 1971, pp. 3-31

[K] Kirby, R.C.: A calculus for framed links in $S^{3}$. Invent. Math. 45, 35-56 (1978)

[KM1] Kirby, R., Melvin, P.: Evaluations of the 3-manifold invariants of Witten and ReshetikhinTuraev for $\operatorname{sl}$ (2. C). In: Geometry of Low-Dimensional Manifolds. London Math. Soc. Lect. Note Ser. 151, Cambridge: Cambridge Univ. Press 1990, pp.473-545

[KM2] Kirby, R., Melvin, P.: The 3-manifold invariants of Witten and Reshetikhin-Turaev for sl(2, C). Invent. Math. 105, 473-545 (1991)

[KM3] Kirby, R., Melvin, P.: Dedekind sums, $\mu$-invariants and the signature cocycle. Invent. Math. (to appear)

[MH] Milnor, J., Husemoller, D.: Symmetric Bilinear Forms. Berlin, Heidelberg, New York: Springer 1973

[RT] Reshetikhin, N.Yu., Turaev, V.G.: Invariants of 3-manifolds via link polynomials and quantum groups. Invent. Math. 103, 547-597 (1991)

[W] Witten. E.: Quantum field theory and the Jones polynomial. Commun. Math. Phys. 121, 351-399 (1989)

Communicated by N.Yu. Reshetikhin 
